\title{
Analyse des causes de surestimation de l'eau corporelle de la chèvre par l'espace de diffusion de l'urée
}

\author{
P Bas, P Schmidely, D Sauvant, \\ P Morand-Fehr, A Rouzeau
}

INRA, station de nutrition et alimentation de I'INA-PG, 16. rue Claude-Bernard, 75231 Paris Cedex 05, France

\begin{abstract}
Summary - Study of the causes of overestimation of body water in the dairy goat from urea injection. Difference between urea space and empty body water (EBW) in late lactating goats and dry non pregnant goats is equal to $1.7 \mathrm{~kg}(\mathrm{~s}=1.58)$. Compartmental analysis of urea exchange in the body is used to predict this difference.
\end{abstract}

L'espace de diffusion de l'urée semblerait être un bon estimateur de l'eau de corps v'de chez la chèvre (Bas et al, 1988). Certaines études sur chevreaux (Schmidely et al, 1990) et sur vaches (Agabriel et al, 1990) ayant souligné certaines limites de cette méthodologie, nous en avons étudié quelques facteurs de variations.

Matériel et méthodes - Vingt-deux chèvres (15 de race alpine et 7 Saanen), âgées de 2 à 7 ans, reçoivent une injection d'urée dans la veine jugulaire à raison de $130 \mathrm{mg} / \mathrm{kg}$ de poids vif (PV) selon les conditions décrites par Bas et al (1988). Douze chèvres sont taries non gestantes (lot $T, 17,1$ ( $s=9,1 \%$ ) lipides / poids vif) et 10 autres (lot L, $12,6 \%$ lipides / poids vif) sont au $5^{\ominus}$ mois de lactation (production $1,6 \mathrm{~kg} / \mathrm{j}, s=0,7$ ). La ration complète du lot $T$ est constituée de foin de prairie permanente $(51,7 \%$ de la matière sèche, MS), de pulpes de betteraves $(27,6 \%)$ el d'orge $(20,7 \%)(0,90$ UFL; $93 \mathrm{~g}$ de PDIE et $78 \mathrm{~g}$ de PDIN/kg MS). La ration du lot $L$ est à base de foin de luzerne $(30,5 \% / M S)$, de pulpes de betteraves
$(31,1 \%)$ et d'un concentré $(38,4 \%)$ riche, soit en amidon (A), soit en parois cellulaires (B) (rations LA et LB) (LA : 0,86 UFL; $95 \mathrm{~g}$ PDIE et $95 \mathrm{~g}$ PDIN $/ \mathrm{kg} \mathrm{MS}$; LB : 0,86 UFL, $108 \mathrm{~g}$ PDIE et $101 \mathrm{~g}$ PDIN). Les urémies après injection (Ut) sont ajustées par le modèle à 2 compartiments Ut-Uo (urémie basale) $=A_{1} e^{-k_{1} t}+A_{2} e^{-k_{1} t}$. L'espace de diffusion de l'urée (EUA) est égal au rapport de la quantité d'urée injectée (Q1)/A2. Les volumes des 2 compartiments, central d'injection (VC) et périphérique (VP) ont été calculés selon les indications de Atkins (1973). Les chèvres sont abattues dès la fin des prélèvements de sang. L'eau du corps vide (ECV) est déterminée par broyage puis lyophilisation. Les facteurs de variations de DUC (DUC = EUA-ECV) ont été déterminés par régression multiple progressive ascendante.

Résultats et discussion - L'ECV ne diffère pas significativement entre les 2 lots $(24,3 \mathrm{~kg}, s=5,6)$ même lorsqu'elle est rapportée au poids du corps vide ( $P C V)(57,8, s=7,3)$. Les 4 paramètres du modèle des cinétiques ne se distinguent pas entre lots $\left(A_{1}=302 \mathrm{mg} /\right.$, 
$s=103 ; A_{2}=27,3 \mathrm{mg} / \mathrm{l}, s=32,2 ; \mathrm{k}_{1}=$ $0,211 \mathrm{~min}^{-1}, s=0,056 ; k_{2}=0,0018$, $s=0,0018)$. EUA $(26,0, s=6,1)$ surestime ECV de $6,9 \%$ environ (ECV $(\mathrm{kg})=0,933(s=0,0118)$ EUA $(\mathrm{kg})$, $\mathrm{ETR}=1,48 \mathrm{~kg}, R=0,998)$. La surestimation (DUC $=1,68 \mathrm{~kg}, s=1,58$ ) est significativement liée à VC $(12,6 \mathrm{~kg}$, $s=3,1, R=0,483, P<0,05)$. Par contre, DUC n'est pas significativement liée à $V P$, à Uo, aux produits $V E \times$ Uo et $V P \times$ Uo, ou pour le lot $L$, à la production laitière. La combinaison de VE et de ECV/PV permet d'améliorer significativement l'explication de la variance de la surestimation (DUC $=0,283$ VE $-0,094$ $\mathrm{ECV} / \mathrm{PCV}+3,56 ; R=0,64 ; \mathrm{ETR}=1,30 \mathrm{~kg}$ ) L'introduction de $A_{2}$ et de l'eau des contenus digestifs/PV (ECO/PV), comme variaoles explicatives supplémentaires, réduit I'ETR à $0,82 \mathrm{~kg}$.

En ne tenant compte que des critères mesurables sans abattage, $V C$ et la différence DPDI = PDIE - PDIN ingérée la veille de l'injection peuvent expliquer $30 \%$ de la variation de DUC
(DUC $=0,220$ VC $+0,09$ DPDI - 1,87; $R=0,58, \operatorname{ETR}=1,38$ ).

En conclusion, la surestimation de I'ECV par l'espace de diffusion de l'urée n'est pas significativement influencée par les stades physiologiques étudiés. Elle est plus importante pour les animaux présentant un état d'engraissement et un volume du compartiment central (VC) plus importants. En outre, la distribution d'un régime plus pauvre en azote fermentescible semble favoriser la surestimation peut-être parce que le flux de recyclage de l'urée dans le tube digestif est plus élevé que celui de son excrétion rénale.

Agabriel J, Decourtenay MB (1990) Reprod Nutr Dev (suppl 2), 249S-250S

Atkins (1973) In: Modèles et compartiments multiples pour les systèmes biologiques. Gauthier-Villars, Paris

Bas $P$, Morand-Fehr $P$, Sauvant $D$, Hervieu J (1988) Reprod Nutr Dev 28 (suppl 1), 185-186

Schmidely P, Bas P, Rouzeau A (1990) $R e$ prod Nutr Dev (suppl 2), 251 s-252s 